\title{
Determinación de la sensibilidad por CIM y método de difusión en placa de 13 antibióticos frente a 55 cepas de Salmonella Typhimurium
}

\author{
Juan Silva, Nelson Herrera, Rodolfo Urdanivia, Mariana Jofré, Hernán Sagua.
}

Grupo de Microbiología y Parasitología. Departamento Ciencias de la Salud. Universidad de Chile, Sede Antofagasta.

Las salmonellas son bacilos Gram negativos. aerobios $y$ anaerobios facultativos, cuya principal característica es que son patógenos para el hombre y los animales por vía oral. En las salmonellosis se pueden producir tres tipos de enfermedad: Fiebres intestinales (tifoidea), septicemias y gastroenteritis agudas. Las formas mixtas son frecuentes en la clínica humana, (Jawetz y col., 1977).

A nivel mundial se ha observado que la tifoidea ha disminuido, en cambio las infecciones por otras salmonellas ha aumentado en forma sostenida, principalmente como brotes epidémicos estacionales e intrahospitalarios, (Prado y col., 1974; Grados, 1973).

Una de las especies que con mayor frecuencia se ha aislado en estos episodios ha sido Salmonella typhimurium, de amplia distribución en la naturaleza, constituyendo el fecalismo humano y animal la principal fuente de contaminación, (Aburto, 1965; Pinto, 1965; Castillo y col., 1973; Rolfo y col., 1973; Lobos y col., 1976).

En el niño ocasiona cuadros severos de gastroenteritis con período de incubación entre 4 y 48 horas, adoptando formas mixtas de septicemias o meningitis, además de una gran resistencia a la mayoría de los antimicrobianos de uso habitual en la terapéutica para bacilos Gram negativos entéricos, (Lobos y col., 1973; Salcedo y col., 1969; Lynch y col., 1975).
Frente a estos antecedentes, y debido a la aparición de un incremento de las infecciones por $S$. typhimurium en el Servicio de Pediatría del Hospital Regional Dr. L. Guzmán de Antofagasta al finalizar 1978, hemos querido evaluar la susceptibilidad de las cepas aisladas, frente a diversos antibióticos; comparando a su vez la susceptibilidad o resistencia mediante los métodos de dilución seriada en agar y el de difusión del disco.

\section{MATERIAL Y METODO}

Se midió la susceptibilidad de 55 cepas de Salmonella typhimurium, provenientes de pacientes del Servicio de Pediatría del Hospital Regional Dr. L. Guzmán de Antofagasta, aisladas entre septiembre de 1978 y enero de 1979.

Cuarenta y tres cepas se obtuvieron de coprocultivos y 12 de hemocultivos, siendo identificados desde el punto de vista bioquímico y serológico (Edwards y col. 1972) en el Laboratorio Central de dicho Hospital y en los Laboratorios del Grupo de Microbiología de la Universidad de Chile, Sede Antofagasta.

Para la medición de susceptibilidad a los antimicrobianos se utilizaron el método de dilución seriada en placa y el difusión del disco, según Ericsson y Sherris (1971). Los antibióticos ensayados fueron del grupo de: Aminoglu- 
cósidos (neomicina, gentamicina, estreptomicina, sisomicina, aminosidina y kanamicina); Cloramfenicol (Cloramfenicol) Penicilinas (ampicilina y amoxicilina); Tetraciclinas (tetraciclina); Rifamicinas (rifampicina). En tanto que los quimioterápicos fueron: Sulfonamidas (sulfametoxazole); Furantoínas (furazolidona).

En la determinación de concentración inhibitoria mínima (CIM), por el método de dilución en placa, el umbral de sensibilidad correspondió a $8 \mathrm{mcg} / \mathrm{ml}$ para todas las sustancias ensayadas. En relación a los sensidiscos la susceptibilidad quedó indicada por la zona de inhibición del crecimiento alrededor del disco de potencia única.

\section{RESLLTADOS}

De las 55 cepas estudiadas, el $72,7 \%$ fue sensible a rifampicina con dosis mínima inhibitoria a concentraciones iguales o inferiores a $8 \mathrm{mcg} /$ $\mathrm{ml}$, mientras que para los Aminoglucósidos, Cloramfenicol y Tetraciclinas a esta misma concentración la sensibilidad de las cepas fue de sólo $11,2 \% ; 11,0 \%$ y $14,5 \%$ respectivamente. Las penicilinas de amplio espectro (ampicilina $y$ amoxicilina) presentan CIM muy altos y sólo el $2,7 \%$ de las cepas fue sensible a concentraciones iguales o inferiores a $8 \mathrm{mcg} / \mathrm{ml}$.

De los dos quimioterápicos ensayados, las cepas fueron sensibles en $3,6 \%$ a las Sulfonamidas y en el 98,0\% a las Furantoínas a CIM de concentraciones iguales o inferiores a $8 \mathrm{mcg} / \mathrm{ml}$ Tabla 1.

En la Tabla 2 se presentan los resultados de la determinación de la susceptibilidad a los agentes antimicrobianos ensayados de las 55 cepas por el método de difusión del disco, destacando la alta sensibilidad de rifampicina (discos de $50 \mathrm{mcg} / \mathrm{ml}$ ) con $87,3 \%$ y la furazolidona (Disco de $100 \mathrm{mcg} / \mathrm{ml}$ ) con $98,0 \%$. Los otros antibióticos y quimioterápicos probados se muestran con altos porcentajes de resistencia.

\section{DISCUUSION}

Ampliamente reconocida $y$ determinada la multirresistencia de $S$. typhimurium a los antibióticos y quimioterápicos a nivel nacional y mundial, se plantea como un problema terapeútico cada vez de más difícil solución, puesto que los agentes antimicrobianos habitualmente útiles frente a las salmonellosis muestran una tendencia creciente de menor sensibilidad a cepas de $S$. typhimurium aisladas en distintas áreas geográficas.

La incidencia de la resistencia a grupos de antibióticos con estructura quimica semejante ha aumentado considerablemente en los últimos años. En el caso de gentamicina (aminoglucósidos), Lobos y col., loc. cit. encontró que el $54,35 \%$ de 46 cepas presentaban dosis minima inhibitoria menor o igual a $6,25 \mathrm{mcg} /$ $\mathrm{ml}$; mientras que en nuestro estudio, el mismo ant ibiótico fue sensible en sólo el 9,1\% de las cepas a C:IM menor o igual a $8 \mathrm{mcg} / \mathrm{ml}$. Frente a cloramfenicol y tetraciclina el comportamiento de nuestras cepas fue muy semejante al observado en los aminoglucósidos (Tabla 1).

La susceptibilidad a Penicilina de amplio espectro (ampicilina y amoxicilina) de las 55 cepas, se determinó que sólo el $2,7 \%$ de ellas respondía a una CIM de $8 \mathrm{mcg} / \mathrm{ml}$.

Frente a los quimioterápicos, sulfametoxazole y furazolidona, encontramos que el 3,6\% y $98,0 \%$ de los cultivos examinados eran sensibles a dosis inhibitorias mínimas menores o iguales a $8 \mathrm{mcg} / \mathrm{ml}$, lo que concuerda con 10 determinado por otros autores nacionales, (Lynch y col. 1969; Lobos y col., loc. cit.) para los mismos ant imicrobianos.

Respecto a la determinación de la susceptibilidad de las 55 cepas de $S$. typhimurium a los agentes antimicrobianos por el método de difusión del disco-técnica de uso habitual y obligada en los laboratorios de hospital, más aún en el caso de este tipo de salmonellosis- creemos que se muestra bastante confiable con el empleo de discos de alta potencia y la medición de los diámetros de la zona de inhibición en forma uniforme. Siendo valioso aplicar un control de calidad periódico con cepas de reconocida susceptibilidad estable durante largos períodos, de tal forma que se pueda asegurar la obtención de resultados correctos.

Se observó total concordancia entre los porcentajes de sensibilidad que respondían a una CIM de $8 \mathrm{mcg} / \mathrm{ml}$ y los porcentajes obtenidos por medición de los diámetros de los halos de in- 
SALMONELLA TYPHIMURIUM. SUSCEPTIBILIDAD DE 55 CEPAS A

DROGAS ANTIMICROBIANAS DETERMINADA POR CIM (en mcg/ml). ANTOFAGASTA. CHILE, 1979

\begin{tabular}{|c|c|c|}
\hline \multirow{2}{*}{$\begin{array}{l}\text { Antimucrobiano por } \\
\text { estructura química }\end{array}$} & \multicolumn{2}{|c|}{$\begin{array}{c}\text { Susceptibilidad } \\
\text { Porcentaje } 8 \mathrm{mcg} / \mathrm{ml}\end{array}$} \\
\hline & $\begin{array}{c}\text { Sensibles } \\
\%\end{array}$ & $\begin{array}{c}\text { Resistentes } \\
. \%\end{array}$ \\
\hline \multicolumn{3}{|l|}{ Antibióticos } \\
\hline Aminoglucósidos & $11 ; 2$ & 88,8 \\
\hline Cloramfenicol & 11,0 & 89,0 \\
\hline Tetraciclinas & 14,5 & 85,5 \\
\hline Penicilinas ${ }^{2}$ & 2,7 & 97,3 \\
\hline Rifamicinas & 72,7 & 27,3 \\
\hline \multicolumn{3}{|l|}{ Quimioterápicos } \\
\hline Sulfonamidas & 3,6 & 96,4 \\
\hline Furantoínas & 98,0 & 2,0 \\
\hline
\end{tabular}

hibición de los discos de antibióticos y quimioRESUMEN terápicos ensayados (Tabla 2).

Tabla 2

SALMONELLA TYPHIMURIUM. SUSCEPTIBILIISAD

IJE 55 CEPAS A IDROGAS ANTIMICROBIANAS DETERMINAI)A POR SENSIDISCOS. ANTOFAGASTA CHILE. 1979

\begin{tabular}{|c|c|c|c|}
\hline \multirow{2}{*}{$\begin{array}{l}\text { Agente } \\
\text { antimt- } \\
\text { crobiana }\end{array}$} & \multirow{2}{*}{$\begin{array}{l}\text { Potencia } \\
\text { deldisco } \\
(\mathrm{mcg} / \mathrm{ml})\end{array}$} & \multicolumn{2}{|c|}{$\begin{array}{l}\text { Susceptibilidad } \\
\text { (Disco único) }\end{array}$} \\
\hline & & $\begin{array}{c}\text { Sensibles } \\
\%\end{array}$ & $\begin{array}{c}\text { Resistentes } \\
\%\end{array}$ \\
\hline Gentamicina & 10 & 9,1 & 90,9 \\
\hline Kanamicina & 30 & 10,9 & 89,1 \\
\hline Sisomicina & 10 & 10,9 & 89,1 \\
\hline Estreptomicina & 10 & 1,8 & 98,2 \\
\hline Neomicina & 30 & 12,7 & 87,3 \\
\hline Aminosidina & 50 & 16.3 & 83,7 \\
\hline C:loramfenicol & 30 & 7.3 & 92.7 \\
\hline Ietraciclina & 30 & 10.9 & 89,1 \\
\hline Ampicilinat & 25 & 5,5 & 94,5 \\
\hline Amoxicilina & 60 & 3.6 & 96.4 \\
\hline Rifampicina & 50 & 87,3 & 12,7 \\
\hline Co-trimoxazol & 25 & 5,5 & 94.5 \\
\hline Furazolina & 100 & 98,0 & 2,0 \\
\hline
\end{tabular}

Finalmente, creemos que la vigilancia permanente y sistemática de la susceptibilidad de S. typhimurium a las sustancias antimicrobianas debe practicarse en todo hospital, de tal forma que se puedan adecuar los tratamientos a las dosis y a las drogas de mayor utilidad.
Se investigo la CiM y el antibiograma por sensidisco a Neomicina, Amoxicilina, Cloramfenicol, Sulfametoxazol. Ietraciclina, Kanamicina, Ampicilina. Estreptomicina, Rifampicina, Gentamicina, Sisomicina, Aminosidina y Furazolidona, en 55 cepas de Salmonella typhimurium de pacientes pediátricos internados en el Hospital Regional de Antofagasta.

Se determinó una alta resistencia a los antibióticos por ambos métodos de las cepas en estudio, con CIM superiores a $8 \mathrm{ncg} / \mathrm{ml}$, excepto para rifampicina y furazolidona en que se obtienen CIM inferiores a $8 \mathrm{mcg} / \mathrm{ml}$ en el $72,7 \mathrm{y}$ $98 \%$ de las cepas respectivamente.

Se observó amplia concordancia con el método de difusión en placas, utilizando sensidiscos de concentración única para las mismas drogas en estudio.

\section{SLMMARY}

The MIC: and the antibiogram were investigated by sensidisc with Neomycin, Amoxyline, Chloramphenicol, Sulfamethoxazole. Tetracycline, Kanamycin, Ampicillin, Streptomycin, Rifampycin, Gentamycin, Sisomycin, Aminosidine, and Furazolidone in 55 strains Salmonella Typhimurium. They were sbtained from intern pediatric patients in the Regional lospital of Antofagasta.

$A$ high resistance of the stranis in study to the antibiotics was demostrated by both methods with MIC superior to $8 \mathrm{mcg} / \mathrm{ml}$, except for Rifampicyn, and Furazolidone in which MIC inferior to $8 \mathrm{mcg} / \mathrm{ml}$ in $72.7 \%$ and $98 \%$ of the strans were respectively obtained.

An extensive concordance was observed with the diffusion method in plates, Sensi-dises of single dose were used for the same drugs in study. 